nen grosso modo konservative Kräfte, die in Richtung eines militanten Antikommunismus gingen und denen eindeutig keine Sympathien für den sowjetischen Staat nachgesagt werden konnte.

\title{
1.7 Der realistische Blick auf die sowjetische Außenpolitik unter Truman nach dem Ende der Anti-Hitler-Koalition
}

Im Folgenden soll in aller Kürze auf den Verlauf des entstehenden Kalten Krieges eingegangen werden. Hierbei muss es ausreichen, auf einige entscheidende Etappenschritte der US-amerikanischen Politik sowie auf Stellungnahmen einflussreicher US-Diplomaten einzugehen, da die komplexe Geschichte der Ost-West-Blockkonfrontation vorausgesetzt werden kann. ${ }^{68}$ Dass mit dem Ende des siegreichen militärischen Kampfes gegen das nationalsozialistische Deutschland die systembedingte Auseinandersetzung mit dem existenziellen Gegner, d.h. dem sowjetischen Totalitarismus, weiterging, war nicht zuletzt den Kommunismusexperten im State Department um Kennan bewusst. Die Ost-West-Konfrontation war in den Jahren der Anti-Hitler-Koalition nur ruhiggestellt und spitzte sich nach 1945 immer mehr zu.

Die Kapitulation Deutschlands und das damit einhergehende Auseinanderbrechen der siegreichen Koalition zwischen den demokratischen Westmächten und der stalinistischen Sowjetunion hatte unter anderem zur Folge, dass mehr und mehr die völlig entgegengesetzten Gesellschaftsordnungen sowie politischen Ziele und ideologischen Vorstellungen der ehemaligen Partner ins öffentliche Bewusstsein drang. Obwohl

for Pacific Relations und war seit 1939 der Direktor des »Chinese History Project«an der Columbia University. Nachdem er sich schon in den 1920er-Jahren mit einigen Veröffentlichungen über China hervortat und nach der Verhaftung durch die Nazis in mehreren Konzentrationslagern »durch die Hölle des totalitären Terrors gegangen« war, emigrierte er 1934 vor dem Nationalsozialismus. Unter dem Pseudonym Klaus Hinrichs veröffentlichte Wittfogel in London den Roman Staatliches Konzentrationslager VII. Weithin bekannt wurde er insbesondere durch den Klassiker der Totalitarismusforschung Oriental Despotism (New Haven [NY] 1957; dt. Ausg.: Die Orientalische Despotie. Eine vergleichende Untersuchung totaler Macht, Köln 1962). Ein weiterer prominenter Linksintellektueller, der vom amerikanischen Staat weiter beschäftigt wurde, war Herbert Marcuse, der nach der Auflösung des OSS von 1945 bis 1953 vom State Departement als sogenannter Principal research analyst for Germany übernommen wurde. In diesem Zusammenhang legte er als Leiter einer Forschungsgruppe einer Spionageabwehrabteilung des amerikanischen Außenministeriums in Washington eine materialgesättigte Analyse der internationalen Entwicklungspotenziale des kommunistischen Systems vor; hierbei kam er zu dem Ergebnis, dass die kommunistischen Parteien in Westeuropa entweder integriert oder bedeutungslos seien und angesichts einer möglichen wirtschaftlichen Krise davon ausgegangen werden könnte, dass nicht ein kommunistischer Umsturz zu erwarten sei, sondern ein neuer Faschismus. Im Rahmen seiner Tätigkeiten für das State Departement nutzte Marcuse vorhandene Freiräume, um mit Vorarbeiten für sein späteres Buch über den Sowjetmarxismus zu beginnen. Das Forschungsergebnis erschien unter dem Titel Soviet Marxism. A Critical Analysis 1958 in New York (dt. Ausg.: Die Gesellschaftslehre des Sowjetischen Marxismus, Neuwied/Berlin 1964). Interessanterweise bedankte sich Marcuse in dem Buch ausdrücklich bei dem Direktor des Russian Research Center, William L. Langer, der ihm die Möglichkeit für Recherchen in der Institution für den Forschungsband bot. Langer war Marcuses höchster Vorgesetzter als Leiter der OSS-Behörde während der Mitarbeit in der R\&A.

68 Vgl. exemplarisch die beiden Standardwerke von Wilfried Loth, Die Teilung der Welt: Geschichte des Kalten Krieges 1941-1955, München 1987 und Bernd Stöver, Der Kalte Krieg 1947-1991. Geschichte eines radikalen Zeitalters, München 2007. 
in einigen Teilen der westlichen Staaten die Massenverbrechen in der Sowjetunion und die Politik Stalins, die in dem deutsch-sowjetischen Nichtangriffspakt von 1939 gipfelte, vergessen wurden, bewertete die Regierungsmannschaft Trumans die Sowjetunion wesentlich nüchterner. Truman vertrat eine Nachkriegspolitik gegenüber Stalin, die in einem klaren Gegensatz zu den Vorstellungen seines Vorgängers stand. Roosevelt erhoffte sich nämlich noch nach dem alliierten Sieg gegen Deutschland eine Fortsetzung der während des Krieges auf Partnerschaft angelegten Politik Stalins. Er glaubte beispielsweise noch auf der im Februar 1945 stattgefundenen Konferenz in Jalta, dass entsprechend der amerikanischen Tradition auch die Sowjetunion eine auf vernünftige Kompromisse angelegte Verhandlungspolitik betreiben würde. Roosevelt, der sich früher keine Illusionen über die wahre Natur des sowjetischen Staates und ihrer Diplomatie machte, vertrat nun die Ansicht, dass Stalin keine außenpolitischen Expansionsziele mehr verfolgen würde, in Zukunft mit den westlichen Staaten freundschaftlich zusammenarbeiten und den Frieden garantieren würde. $\mathrm{Zu}$ diesem Zeitpunkt stand Roosevelt auch unter dem Einfluss von Beratern wie Harry Hopkins und dem ehemaligen US-Botschafter in Moskau, Joseph Davies, die den Standpunkt vertraten, dass die Politik Stalins einer eher menschenfreundlichen Motivation entsprang und ein primäres Ziel der Sowjetunion darin bestehen würde, ein absolut verlässlicher Unterstützer des amerikanischen Präsidenten bei der Realisierung der von ihm angestrebten "Einen Welt« zu sein. Wie auch schon während der kriegerischen Konfrontation mit dem Nationalsozialismus galt gerade für Davies eine Kritik an den tatsächlichen politischen Verhältnissen in der Sowjetunion und an den Herrschaftsmethoden Stalins als unbegründet. ${ }^{69}$

Diese Einstellung teilte Truman nicht. Genau wie noch während des Zweiten Weltkrieges Churchill waren er und seine einflussreichen Berater, die sich unter anderem den Grundsätzen der »Riga-Fraktion« verpflichtet fühlten, sich über die anbahnende sowjetische Gefahr insbesondere für Europa einig. Tonangebende Vertreter der USamerikanischen Administration mussten nicht erst von einem strikten antikommunistischen außenpolitischen Kurs überzeugt werden. In einem Memorandum von Mai 1945 warnte zum Beispiel der aus dem Kreis der Riga-Gruppe kommende Unterstaatssekretär im State Department, Joseph C. Grew, nachdrücklich vor der Sowjetunion, indem er sie mit den totalitären Diktaturen der ehemaligen Achsenmächte Deutschlands und Japans verglich und im Hinblick auf die Gefährlichkeit für die Vereinigten Staaten keine Unterschiede machte. Wie Grew verwies auch der aus der »realistischen Riga-Schule« stammende amerikanische Botschafter in Moskau, Averell Harriman, auf die Gefahr einer Invasion Europas durch die Sowjetunion. ${ }^{70}$

69 Vgl. Joseph Davies, Mission to Moscow, New York 1941. Davis vertrat hier zum Zeitpunkt des deutschen Überfalls auf die Sowjetunion die Ansicht, dass Stalin sich von dem eigentlichen Ziel des kommunistischen Regimes, nämlich der Weltrevolution, im Prinzip verabschiedet hatte und sich auf dem Weg befand, ein normaler kapitalistischer Staat zu werden. Wie Davis zeichnete auch der aus der demokratischen Linken kommende und von 1940 bis 1944 als Vizepräsident fungierende Henry A. Wallace während des Zweiten Weltkrieges ein affirmatives, kritikloses Bild der Sowjetunion. Für Wallace war Stalin ein verlässlicher Partner, dem die westlichen Staaten dabei unterstützen müssten, einen demokratischen Reformkurs einzuschlagen.

70 Siehe Leonid Luks, Geschichte Russlands und der Sowjetunion. Von Lenin bis Jelzin, Regensburg 2000, S. 395 
Für eine zunehmend härtere Gangart gegenüber der Sowjetunion plädierte dann zu Beginn des Jahres 1946 Kennan in seiner Funktion als Stellvertreter Harrimans in Moskau. Kennan war von der Verständigungspolitik Roosevelts gegenüber Stalin in seinen letzten Amtsmonaten alles andere als erfreut und begegnete ihr mit zunehmender Skepsis. Nicht zuletzt durch die ständigen Verletzungen der sowjetischen Staatsführung gegen die Abmachungen der Jalta-Konferenz und durch den allmählich einsetzenden Prozess der Sowjetisierung der osteuropäischen Länder konnte sich Kennan in seiner skeptischen Grundhaltung gegenüber dem kommunistischen Herrschaftssystem unter Stalin bestätigt fühlen. So formulierte er in seinem berühmt gewordenen 8000 Worte umfassenden Langen Telegramm vom 22. Februar 1946 die zukünftig überaus wirkungsmächtigen Prinzipien der Eindämmungspolitik. Auf der Basis seiner früheren Arbeiten aus den 1930er- Jahren über das kommunistische Russland fertigte er eine genaue Analyse der zu erwartenden sowjetischen Außenpolitik an. Aus seiner Wahrnehmung heraus stellte der sowjetische Staat auf der Grundlage der marxistisch-kommunistischen Ideologie auch weiterhin ein »Polizeiregime par excellence« dar, das mit der kapitalistischen Umwelt und den demokratischen Nationen in einer ständigen prinzipiellen Feindschaft lebt. Angesichts einer aus seiner Sicht ideologisch motivierten aggressiven sowjetischen Außenpolitik sprach er sich für eine eindeutige antikommunistische amerikanische Außenpolitik aus, die auch und vor allem die Zusammenarbeit mit den westlichen demokratischen Staaten beinhalten sollte. Im Langen Telegramm beschrieb Kennan die Sowjetunion als eine politische Kraft, »die sich fanatisch zu dem Glauben bekennt, daß es mit Amerika keinen dauernden Modus vivendi geben kann, daß es wünschenswert und notwendig ist, die innere Harmonie unserer Gesellschaft, unsere traditionellen Lebensgewohnheiten und das internationale Ansehen unseres Staates zu zerstören, um der Sowjetmacht Sicherheit zu verschaffen $\ll^{71}$.

Im Gegensatz zu der vielerorts gemachten Übereinstimmung zwischen der nationalsozialistischen und sowjetkommunistischen Außenpolitik bestand Kennan darauf, dass Stalin keineswegs eine sozusagen abenteuerliche Kriegspolitik betreiben würde. Seiner Einschätzung nach hatte die westliche Staatenwelt mit einer überaus variablen, gleichwohl vorsichtigen Diplomatie- und Machtpolitik der Sowjetführung zu rechnen. Ein zentrales Ziel sei die Stabilisierung des eigenen politischen Herrschaftsbereiches sowie der Versuch, die westliche Staatenwelt zu schwächen und untereinander auseinanderzudividieren. Für den »Analytiker des Kalten Krieges« (Detlef Bald), Kennan, bestand für die Regierung der Vereinigten Staaten zweifelsohne die Möglichkeit, die Schwierigkeiten der internationalen Sicherheit zu lösen, »und zwar ohne uns in einen großen militärischen Konflikt zu flüchten $^{72}$. Das Kennan-Telegramm sorgte schnell für Furore in den amerikanischen Regierungsstellen, wodurch sich der Verfasser den Ruf des besten »Kreml-Experten« erwarb. Durch den Marineminister James Forrestal wurde der Bericht kopiert und an die zentralen Stellen des US-amerikanischen Staates versandt. Die implizit formulierte Absage an eine Appeasementpolitik gegenüber der Sowjetunion und den Abmachungen von Jalta sowie die Empfehlung für eine Einheit der westlichen Staatenwelt stieß auf breite Zustimmung in Washington. Seine Durch-

71 Zit. n. der deutschen Übersetzung in Kennan, Memoiren eines Diplomaten, S. 566.

72 Ebd., S. 567. 
schlagskraft besaß der Bericht durch seine scheinbare Eindeutigkeit, nämlich »die Bedrohung durch den Osten enttarnt zu haben «. ${ }^{73}$

Kennan wiederum verstand unter einer erfolgreichen Politik eine komplexe Auseinandersetzung mit der Sowjetunion. Der kommunistische Gegner sollte nicht primär militärisch bekämpft werden, sondern notwendig war seiner Einschätzung nach die politische und ideologische Auseinandersetzung mit dem Kommunismus. Jedenfalls sollte Kennan mit seinem Bericht für lange Zeit nicht nur die Außenpolitik der Vereinigten Staaten in wesentlichen Eckpunkten vorgeben; anders gesagt: Auf der Grundlage von Kennans konzeptionellen Vorschlägen wurde die als Containment-Politik in die Geschichte des Kalten Krieges eingegangene strategische Neuausrichtung der USamerikanischen Regierungspolitik begründet. Die Bedeutung, die Kennan zu diesem Zeitpunkt in der US-Administration erlangt hatte, konnte daran abgelesen werden, dass er zum Leiter der einflussreichen Policy Planning Staff im State Department ernannt wurde. Insbesondere im amerikanischen Außenministerium war dem Bericht Erfolg beschieden, weil man sich hier in seiner nach 1945 allmählich stärker werdenden antisowjetischen Position bestätigt sehen konnte. Auf viele Aspekte des Langen Telegramms ging Kennan im Juli 1947 in einem Aufsatz ein, den er mit Erlaubnis des Außenministeriums unter dem Pseudonym »X« im Foreign Affairs veröffentlichte, das zu den Zeitschriften zählte, in denen der Meinungsbildungsprozess in dem amerikanischen politischen Führungszirkel mitvollzogen wurde. Hier forderte er eine »Eindämmungspolitik« zum Schutze der westlichen demokratischen Staaten gegen den auf Expansion zielenden Kommunismus. ${ }^{74}$

Wie gezeigt, übernahm nach Beendigung des Zweiten Weltkrieges und der Auflösung des OSS-Apparates das State Department nicht nur die sogenannten Deutschlandexperten, sondern auch die Division UdSSR aus der Forschungsabteilung - R\&A -, die durch ihre wissenschaftlichen Studien über den Kommunismus frühzeitig wussten, dass die totalitäre Bedrohung durch die Sowjetunion unter Stalin in den Jahren des gemeinsamen Kampfes gegen den Nationalsozialismus nur auf »Eis« gelegt war. Dass im State Department frühzeitig gewissermaßen die Zeichen auf Sturm standen und die sowjetische Bedrohung als ein ernst zu nehmender Faktor erkannt wurde, dafür waren auch die Kommunismusforscher verantwortlich, die sich bereits während des Zweiten Weltkrieges mit Fragen der Nachkriegspolitik der Vereinigten Staaten beschäftigt hatten. Wie in der existenziellen Auseinandersetzung mit dem Nationalsozialismus wurden auch nach 1945 Intellektuelle und Wissenschaftlicher als politische »Waffe« in der US-Administration eingesetzt - jetzt in zunehmend stärkerem Maße gegen die Sowjetunion. Die Regionalabteilung UdSSR sorgte fortan im State Department dafür, dass die Auseinandersetzung mit dem kommunistischen Russland auch mit wissenschaftlichen Methoden betrieben wurde. Indessen wurden einige wenige ehemalige Mitarbeiter der Division UdSSR des OSS aus dem amerikanischen Staatsdienst entlassen, denen wie beispielsweise beim Wissenschaftler Paul A. Baran Sympathien mit dem kommunistischen Regime Stalins nachgesagt wurde.

73 Detlef Bald, Hiroshima, 6. August 1945. Die nukleare Bedrohung, München 1999, S. 59.

74 George F. Kennan (unter dem Pseudonym »X«), The Sources of Soviet Conduct, in: Foreign Affairs 25 (1946/47), S. 566 ff.; siehe auch zu den näheren Umständen der Veröffentlichung und zur Interpretation des Aufsatzes die Ausführungen in Kennan, Memoiren eines Diplomaten, S. 357-370. 
Die öffentliche Meinung in den Vereinigten Staaten sollte vor allem durch die berühmt gewordene Eiserne-Vorhang-Rede von Churchill im Beisein von Truman am 5. März 1946 in Fulton, Missouri, beeinflusst werden. Durch die Beschwörung der sowjetischen Gefahr sollte insbesondere isolationistischen Ambitionen in den USA entgegengewirkt werden und der prosowjetischen Linken um den ehemaligen Vizepräsidenten und damaligen Handelsminister Henry Wallace das Wasser abgegraben werden. Der Rücktritt des russlandfreundlichen Wallace erfolgte dann im September 1946. Zudem stellte die Rede Churchills ein Plädoyer zur Gründung einer amerikanisch-britischen Allianz dar und mit ihr sollte der amerikanischen Öffentlichkeit signalisiert werden, dass die Stationierung der US-Soldaten in Europa auch zukünftig notwendig wäre. Angesichts des sich weltweit ausbreitenden Kommunismus, der rigorosen sowjetischen Politik in Osteuropa und der sich vermehrenden Anzeichen für eine kommunistische Machtübernahme in Südosteuropa (u. a. Griechenland) und insbesondere in zwei Kernstaaten West- und Südeuropas (Frankreich/Italien) verkündete dann am 12. März 1947 der amerikanische Präsident in seiner Rede vor den beiden Häusern des Kongresses seine als »Truman-Doktrin« in die Geschichte eingegangene Bereitschaft, »die freien Völker zu unterstützen, die sich der Unterwerfung durch bewaffnete Minderheiten oder durch Druck von außen widersetzen ${ }^{75}$. Truman machte unmissverständlich klar, dass die Vereinigten Staaten entschlossen waren, fortan allen vom Kommunismus bedrohten Staaten Hilfe anzubieten. Zum ersten Mal kam hiermit praktisch die Vorstellung zum Tragen, dass "man Eindämmung offensiv betreiben müsse, wenn sie erfolgreich sein solle ${ }^{76}$. Neben Militärhilfe sah dies umfassende amerikanische Wirtschaftshilfe für Europa vor, die in dem nach dem damaligen Außenminister Marshall benannten und vom Kongress genehmigten Marshallplan ihren bedeutenden Ausdruck fand. Mit dem Angebot der US-Regierung angesichts der ihrer Auffassung nach drohenden Gefahr für die Freiheit der eigenen Staatsform, die »freien Völker" gegen die aggressive und expansive Machtpolitik der Sowjetunion zu unterstützen und ihnen beizustehen, war auch eine sichtliche Absage an jede Art von Isolationismus verbunden. Damit sollte auch eine Wiederholung der Fehler nach dem Ersten Weltkrieg vermieden werden. ${ }^{77}$

Die Eindämmungsdoktrin zementierte die Zweiteilung der Welt in einen demokratischen und kommunistischen Staatenblock und war Ausdruck eines amerikanischen Grundkonsenses. Spätestens im Frühjahr 1947 war der antikommunistische Konsens, der sich allerdings von einzelnen Personen und Gruppen in der US-Staatsadministration keinesfalls erst nach dem Ende des Zweiten Weltkrieges herauskristallisieren musste - unter anderem im State Department bei der sogenannten Riga-Gruppe -, zu einem mächtigen Faktor in der Politik Washingtons geworden. Einflussreiche Persönlichkeiten des US-amerikanischen Regierungsapparates wie z. B. Kennan mussten nicht erst durch die konkreten Erfahrungen mit der stalinschen Diplomatie nach dem siegreichen Kampf gegen den nationalsozialistischen Totalitarismus vom eigentlichen

75 Zit. n. Loth, Die Teilung der Welt, S. 160.

76 Stöver, Der Kalte Krieg 1947-1991, S. 23.

77 Ebd., S. 23. 
Wesen des sowjetischen Herrschaftssystems überzeugt werden. Der totalitäre Herrschaftscharakter des Stalinismus stand für sie fest. ${ }^{78}$

\subsection{Die Gründung der CIA und ihre verdeckten Operationen}

Während bereits der als ein weltweites Bollwerk zur Absicherung des amerikanischen Einflusses gedachte Marshallplan wesentlich auf den politischen Vorstellungen von Kennan beruhte, hatte der Direktor des Politischen Planungsstabes im Außenministerium außerdem einen erheblichen Anteil an der Gründung der CIA am 18. September 1947. Die Schaffung des ersten Geheimdienstes der Vereinigten Staaten zu »Friedenszeiten" stand hierbei ganz im Zeichen des antikommunistischen Grundkonsenses in der amerikanischen Führung und des heraufziehenden Kalten Krieges. Kennan wurde hierbei unterstützt von führenden Persönlichkeiten aus dem Außenministerium und dem diplomatischen Dienst wie Charles »Chip« Bohlen und Averell Harriman (also Exponenten der »Riga-Fraktion) sowie John McCloy, Joseph und Stewart Alsop, Walter Lippmann, Dean Acheson, George C. Marshall oder James Forrestal. Sie waren tonangebend in der US-amerikanischen Regierungspolitik und teilten nicht nur die Auffassungen über das Wesen des kommunistischen Regimes, sondern auch die Überzeugung, dass die Vereinigten Staaten eine Vorreiterrolle zu spielen hätten im Kampf der westlichen Demokratien gegen die Sowjetunion und ihre Satellitenstaaten. Sie hingen der für die außenpolitische Strategie im Kalten Krieg maßgeblichen traditionellen Vorstellung an, wonach antidemokratische Mächte auf Dauer eine Bedrohung der gewissermaßen natürlichen Interessen der Vereinigten Staaten darstellten. ${ }^{79}$ Ihr zentrales Thema war die Frage, wie die Übernahme Europas durch die Sowjetunion verhindert werden könne, und hierbei sollte neben dem Marshallplan der CIA eine entscheidende Funktion zufallen.

Mit der Unterschrift unter das Nationale Sicherheitsgesetz (National Security Act) am 26. Juli 1947 schuf Präsident Truman die gesetzliche Grundlage für die Gründung der CIA. Zudem wurde mit dem Gesetz der Nationale Sicherheitsrat (National Security Council, NSC) neugeschaffen, der als Vermittlungsstelle des Weißen Hauses für Entscheidungen des Präsidenten fungieren sollte. ${ }^{80}$ Dies entsprach den macht- und

78 Die amerikanische Öffentlichkeit wurde durch zwei weitere Buchveröffentlichungen über den tatsächlichen Charakter des sowjetischen Totalitarismus unterrichtet: vgl. Victor A. Kravchenko, I Chose Freedom, New York 1946 sowie David Dallin/Boris Nicolaevsky, Forced Labor in Soviet Russia, New Haven 1947. Die beiden Veröffentlichungen unterstrichen ein weiteres Mal, dass es sich beim ehemaligen Bündnispartner Sowjetunion um einen totalitären Polizeistaat handelte, und hoben nicht zuletzt die besondere Rolle des Ceheimdienstes NKWD hervor, durch die die sowjetische Bevölkerung eine extreme Unterdrückung und ganz spezifischen Grausamkeiten ausgesetzt sei. Zudem zeigten beide Veröffentlichungen, dass der NKWD das entscheidende Instrument war, um ein umfangreiches, weitverzweigtes Zwangsarbeitslagersystem mit Millionen von Gefangenen aufzubauen und am Leben zu erhalten und dass der sowjetischen Gesellschaft seinen Stempel aufdrückte. Beide Bücher besaßen innen- und außenpolitisch eine hohe Brisanz, weil es nicht nur der amerikanischen Öffentlichkeit ein weiteres Mal eindrucksvoll bestätigte, dass zwischen dem demokratischen System der Vereinigten Staaten und dem sowjetischen Totalitarismus ein fundamentaler Gegensatz bestand.

79 Stöver, Der Kalte Krieg 1947-1991, S. 21.

80 Weiner, CIA, S. 54. 